\title{
Revision in Cemented and Cementless Infected Hip Arthroplasty
}

\author{
Paolo Cherubino, Marco Puricelli and Fabio D’Angelo*
}

\author{
Department of Biotechnologies and Life Sciences, Section of Orthopedics and Traumatology, University of Insubria, \\ Varese, Italy
}

\begin{abstract}
Infection is a frequent cause of failure after joint replacement surgery. The infection rate after total hip arthroplasty (THA) has been reduced to $1-2 \%$ in the last years. However, it still represents a challenging problem for the orthopedic surgeon.

Difficulty of therapeutic approach, and poor functional outcomes together with length of treatment and overall cost are the main burden of this issue. Even the diagnosis of an infected hip could be challenging although it is the first step of an accurate treatment. At the end, many cases require removing the implants. Afterwards, the treatment strategy varies according to authors with three different procedures: no re-implantation, immediate placement of new implants or a twostage surgery re-implantation.

Based on the most recently systematic review there is no suggestion that one- or two-stage revision methods have different re-infection outcomes.

The two-stage implant-exchange protocol remains the gold standard. It is considered as the most efficacious clinical approach for the treatment of periprosthetic infection, especially in patients with sinus tracts, swelling, extended abscess formation in depth and infection of Methicillin Resistant Staphylococcus Aureus (MRSA), and other multidrug-resistant bacteria as reported in recent consensus documents.
\end{abstract}

Keywords: Hip arthroplasty, infection, two-stage revision, spacer.

\section{INTRODUCTION}

Total hip arthroplasty (THA) is currently one of the most successful surgical procedures ever developed. In the United States nearly 700.000 hip and knee arthroplasties are performed annually, with demand predicted to increase substantially [1].

Infection is a frequent cause of failure after joint replacement surgery: between $7 \%$ and $16 \%$ of THA revisions are carried out for infection $[2,3]$. The infection rate after THA has been reduced to $1-2 \%$ [4] in the last years. However, infection still represents a challenging problem for the orthopedic surgeon. The negative implications are several: high costs and length of treatment, difficulty of therapeutic approach, and poor functional outcomes.

Risk factors for periprosthetic joint infection (PJI) are post-traumatic osteoarthritis, diabetes, obesity, smoke, chronic vascular disease, rheumatoid arthritis, bacteraemia, postoperative wound complications, corticoid therapy, immunosuppressant, malignancy, presence of other infections (cutaneous, urinary tract, respiratory, abdominal, oral or dental infections), chronic liver disease, surgical time, inadequate antibiotic prophylaxis, dislocation, or previous arthroplasty [5, 6].

*Address correspondence to this author at the Department of Biotechnologies and Life Sciences, Section of Orthopedics and Traumatology, University of Insubria, Ospedale di Circolo - Viale Borri, 57 - 21100 Varese, Italy; Tel: 39-0332-278824; Fax: 39-0332-278825;

E-mail: fabio.dangelo@uninsubria.it
Regarding the fixation as risk factor, Engsaeter et al. compared the revision rates due to infection in primary uncemented THA with those of cemented one with antibiotic-loaded cement and to those of cemented without antibiotic cement in 56.275 primary THA [7]. They found that uncemented hip arthroplasties had the same risk of revision due to infection as cemented arthroplasties with antibiotic-loaded cement (ALAC), but reduced risk compared to cemented arthroplasties without antibiotic cement. One possible explanation could be that the cementation per se creates, in some ways, conditions that are conducive to the growth of bacteria following the apparently unavoidable peroperativecontamination. Necrotic bone tissue around the cement, caused by both cement toxicity and heat generation, could be the potential growth medium. Antibiotic in the cement could partly protect against growth of bacteria in this area of low resistance to infection. The insertion of an uncemented hip arthroplasty might cause less tissue necrosis and the antibiotic may therefore not be needed to the same extent.

\section{DIAGNOSING AN INFECTED HIP ARTHROPLASTY}

A complete blood cell count has poor sensitivity for the diagnosis of PJI. C-reactive protein (CRP), erythrocyte sedimentation rate (ESR), and, more recently, interleukin 6 (IL-6) are non-invasive tests that may be used in the preoperative assessment of PJI. These tests are affected by non-infectious conditions, such as neoplasia and connective tissue disease [8].

In a study of 636 patients undergoing knee, hip, shoulder, or spine arthroplasty revision, the sensitivity and specificity 
of the ESR $(>30 \mathrm{~mm} / \mathrm{h})$ and CRP $(>10 \mathrm{mg} / \mathrm{L})$ for the diagnosis of PJI varied according to the joint involved. The ESR sensitivities were $71 \%$ for knee, $47 \%$ for hip, and $16 \%$ for shoulder arthroplasty infection. The ESR specificities for the same joints were $89 \%, 84 \%$, and $98 \%$, respectively. The CRP sensitivities and specificities were $83 \%$ and $79 \%$ for knee, 74 and $78 \%$ for hip, and $42 \%$ and $84 \%$ for shoulder arthroplasty infections, respectively. When both tests (CRP and ESR) were negative, the negative predictive value for hip and knee arthroplasty infections was $94 \%$ compared to $77 \%$ for shoulder arthroplasty infection [9]. In a study evaluating 58 patients undergoing total knee or hip arthroplasty revision, IL-6 was found to have $100 \%$ sensitivity and $95 \%$ specificity with a cut-off of $\geq 10 \mathrm{pg} / \mathrm{ml}$ [10], however it has not yet been adopted for routine use.

Plain radiographs lack sensitivity and specificity in diagnosing septic arthroplasty. Findings such as lucencies can be noted in both septic and aseptic loosening. In early infection, plain radiographs are frequently normal [11]. Although lucencies are common, some may be normal variations relating to surgical technique in the case of component-prosthesis interface, or bone reaction to cement in the case of cement-bone interface. Normal lucencies are often found in the proximal lateral aspect of the stem-cement interface, and a $<2 \mathrm{~mm}$ lucency surrounding the cement mantle running parallel to the stem (which results from a stable fibrous reaction to the cement). Lucencies $>2 \mathrm{~mm}$ in thickness or progression of defects may be indicative of loosening or infection. Well demarcated, progressive areas of lucency at the cement-bone interface may indicate infection or granulomatous disease [12]. Thus, there is a need for chronological comparison of films.

Technetium-methylene-diphosphonate (MDP) bone scan is a sensitive test for prosthetic joint failure but lacks specificity, as it does not differentiate between aseptic and septic loosening. Labelled leukocyte imaging should be well suited for diagnosing the infected joint replacement because white cells usually do not accumulate at sites of increased bone mineral turnover in the absence of infection. Many radionuclide tests were developed, but the procedure of choice for diagnosing PJI remain leukocyte/marrow imaging with sensitivity, specificity, and accuracy of $96 \%, 87 \%$, and $91 \%$, respectively, as recently reported by Love [13]. This test is significantly more accurate than bone (50\%), bone/gallium (66\%), and leukocyte/bone imaging (70\%).

Promising results in terms of sensitivity, specificity and accuracy have been recently documented with 18F-fluorodeoxyglucose positron emission tomography [14,15], but published results are not conclusive.

Regarding the treatment, only in selected cases is possible to treat infection with a debridement and component retention procedure: infection eradication rates in the literature vary widely, from 18 to $90 \%$, and it strictly depends on rigorous patient selection criteria $[16,17]$.

Except in these particular and restricted indications, the control of the infection requires removing the implants and all the pathologic tissues. Afterwards, the treatment strategy varies according to authors with three different procedures: no re-implantation, immediate placement of new implants or a two-stage surgery re-implantation $[18,19]$.

\section{ONE-STAGE REVISION (DIRECT EXCHANGE)}

One-stage revision or direct exchange arthroplasty has obvious advantages in the management of infected THA. With one major procedure, the patient is exposed to lower, cumulative perioperative risk. There are also benefits both financially and in terms of resource allocation [11,20].

Some old studies regarding one-stage revision report mean infection-free rates of $80 \%$ (without antibiotic loaded cement) and $82 \%$ (with antibiotic loaded cement) [21-23].

In 2000, Jackson and Schmalzried conducted a literature review to determine factors associated with a successful outcome. Twelve studies, including a total of 1.299 infected THA, were assessed. While antibiotic-impregnated cement was used in $99 \%$ of cases, there were wide variations in antibiotic choice, administration and duration. The average time of follow-up was 4.8 years.

Overall infection-free rate was $83 \%$ at final follow-up. Factors associated with success were: absence of wound complications after the initial THA, good general health, sensitive Staphylococcus or Streptococcus saprophyticus. and organism sensitive to the antibiotic in the cement. Factors associated with poor outcome were: poly-microbial infection, gram-negative organisms, especially pseudomonas and MRSA, and group D Streptococcus. The authors suggested that using cementless implants or bone graft may be a contraindication [24].

Other recent studies reported infection-free rate of 83.3\%-93.7\% with either cementless [25] or combination of cemented-uncemented or hybrid fixation [26] in one stage revision. All authors emphasize that resection should be large and meticulous.

There are some concerns about the addition of antibiotics to cement as they reduce the mechanical properties if the total amount used is more than the $10 \%$ of the weight. Moreover not all antibiotics can be used because they have to be available in powder form, be water-soluble and be thermostable: the most commonly used are gentamicin, clindamycin, vancomycin, tobramycin, aztreonam, ampicillin and ofloxacin [27, 28].

An interesting option is represented by allograft bone impregnated with high loads of antibiotics using special incubation techniques. This antibiotic-loaded compound has some advantages if compared with antibiotic loaded acrylic cement (ALAC): better storage capacities and better pharmacokinetics that provides a prolonged release with higher local concentration. Another theoretical advantage is the possible synergy in restoring the bone stock offered by the graft. This antibiotic loaded graft may be used in combination with uncemented implants for improved long term results and easy removal in case of a failure. Winkler $e t$ al. [29] reported 37 cases treated with this surgical option using cancellous particulated graft mixed with vancomycin, either with or without tobramycin, and demonstrated an eradication rate of $92 \%$ after a follow-up period of 4.4 years: five of the successfully treated patients were MRSA infection.

Consensus recommendations for one-stage exchange suggest that it should only be considered where there is minimal soft tissue damage and where less virulent 
organisms are involved. Ideally, the causative agent should be known, and treatment should be commenced preoperatively [18].

In a recent prospective study Klouche et al. included 84 patients, all diagnosed of infected THA who had prosthesis replacement. A one-stage exchange was performed in 38 cases and a two-stage procedure in 46 cases. A two-stage procedure was decided in the case of important bone loss or unidentified germ. Postoperatively, patients received intravenous antibiotics (six weeks), then oral antibiotics (six weeks). The main evaluation criterion was the rate of infection eradication at 2 years minimal follow-up. If new infection was suspected, a hip aspiration was performed to determine whether it was non-eradication (same germ) or a new re-infection (other germ), which was not considered as a failure. The initial infection was cured in 83 out of 84 patients $(98.8 \%), 38(100 \%)$ for the one-stage group and 45 $(97.8 \%)$ for the two-stage group. Eighty out of $84(95.2 \%)$ patients were infection free, all patients $(100 \%)$ of the onestage group and 42 patients $(91.3 \%)$ of two-stage group. This interesting study demonstrated that, if precise selection criteria were respected, a high success rate in THA replacement for infection may be achieved with a one-stage procedure [19].

\section{TWO-STAGE REVISION}

Two-stage revision has been reported to produce the best results, with an infection eradication rate higher than $90 \%$ [30]. The principles of two-stage revision are the removal of all prosthesic components, including cement, with radical debridement of infected tissue and bone. Local antibiotics, administered with the use of an antibiotic-loaded cement spacer, and systemic antibiotics are used in conjunction. Reimplantation is conducted at 6 to 12 weeks and may be altered depending on multiple factors. Several questions remain, particularly around the timing and the duration of antibiotic administration, the appropriate use of articulating spacers and the timing of reimplantation [31]. Two-stage revision is often considered essential for more virulent infections, as those sustained by Pseudomonas saprophyticus.

The use of antibiotic-loaded cement spacers shaped to mimic a femoral prosthesis has spread widely, and various series with good results are reported [30]. The spacer has both a biological and a mechanical function. A welldesigned spacer releases locally effective dose of antibiotic, which complements the surgical debridement by preventing colonization of bacteria on the spacer surface and complements the systemic antibiotic therapy in eradicating the infection [32]. Furthermore, the spacer provides partial joint mobility and function, helps preserve bone stock, limits the formation of scar tissue, and maintains soft tissue length, thus making the secondary implantation procedure easier and faster [33] and improving the functional outcome [34].

The main disadvantage is the requirement of an extra hospital admission and a further major surgical procedure, however, means that a two-stage revision may cost $70 \%$ more than a one-stage revision [35]. ALAC spacers are classified as static or articulating spacers. Static spacers consist of a block or beads of antibiotic-impregnated cement, left within the dead space after implant removal. Articulating spacers consist in anantibiotic cement prosthesis supported by a metal endoskeleton, and can also be divided into 3 groups: handmade (Fig. 1), molded or preformed ones (Fig. 2).

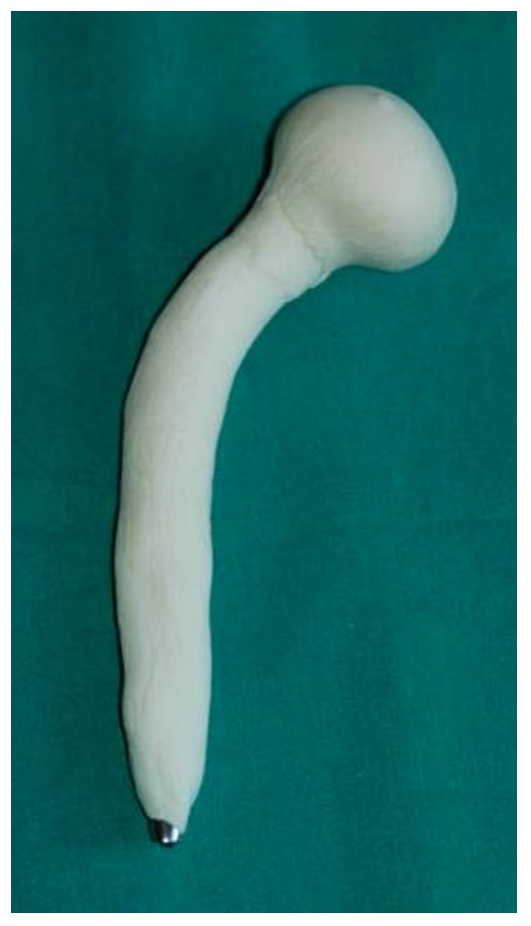

Fig. (1). Example of hand-made spacer.

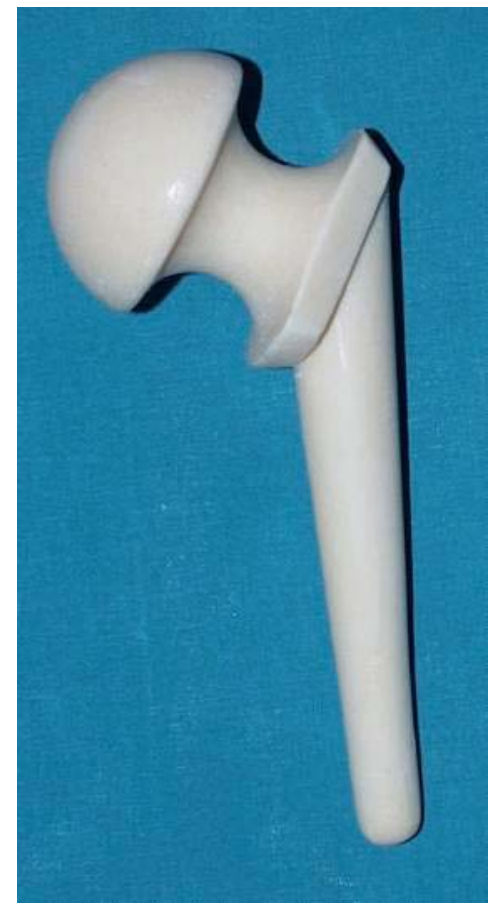

Fig. (2). The Spacer-G/InterSpace.

There are some concerns about these different spacers in terms of incidence of mechanical complications as breakage or dislocation, and also unpredictable antibiotic release.

After hip spacer implantation other than reinfection or infection persistence Jung et al. [36] reported several complications. The study included 88 hand-made spacers in 82 patients. At a mean follow-up of 54 months (7-96), the overall complication rate was $58.5 \%$. Spacer dislocation 
occurred in $17 \%$, spacer breakage in $10.2 \%$ and femoral fractures in $13.6 \%$. The dislocation rate after second stage reimplantation was $23 \%$.

Cabrita et al. [37] presented 68 infected THA treated with two-stage revision. In this study he compared the use of a handmade vancomycin-loaded spacer in 38 patients with a control group of 30 patients where no spacer was used between the two stages. At an average follow-up of seven years (5-11.5 years) the infection-free rate was $66.7 \%$ in the control group and $89.1 \%$ in the group with the spacer. Six mechanical complications were described in the group of the spacers, such as dislocation, breakage and intrapelvic migration.

The PROSTALAC system (DePuy, Warsaw, IN, USA), developed by Duncan et al. [33], is an example of the molded one. In the first trial with the PROSTALAC, Duncan et al. [33] reported three cases of dislocations among 15 patients. In a recent report of 118 hips treated with PROSTALAC, six dislocations occurred [38], showing that experience acquired with this method may diminish such complication.

Spacers using a femoral component as the endoskeleton with a metal-on-polyethylene bearing offer several proposed advantages: they are superior at restoring offset and length; antibiotics can be tailored to the infecting organism and they may confer superior functional results and allow longer periods of use before exchange.

The potential drawback of these techniques is the introduction of the polyethylene liner and a metallic head in an infected site, in addition to the increased surgical time required for its preparation $(60-90 \mathrm{~min})$. Moreover, the polyethylene liner needs to be cemented to the acetabulum, thus making very easy increasing the acetabular bone loss at its removal.

A recent series of 28 patients treated with PROSTALAC insertion awaiting two-stage revision has been published by Scharfenberger et al. in 2007. They have reported two patients that required repeated PROSTALAC insertion for infection persistence and two additional patients that had positive 48-hour cultures post-second stage, treated with additional intravenous antibiotics. Post-second-stage prosthesis retention was in all four patients for up to 4 years after second stage surgery. Patients who have retained the PROSTALAC implant as a semi-permanent implant are also clinically clear of infection. Their results shows resolution rates comparable with those reported in the literature, suggesting that the PROSTALAC is an excellent alternative to simple cement spacers for the treatment of periprosthetic infection. Furthermore these patients were compared to patients awaiting THA and those six months post THA. Western Ontario McMaster (WOMAC) score and Harris Hip Score (HHS) were significantly better than those awaiting THA but less than early primary THA. While the long-term durability of such systems remains to be seen, this type of spacer offers superior functional performance [39].

Another data about the PROSTALAC system were reported by Masri et al. [40] who retrospectively reviewed 29 patients who underwent two-stage revision using cementless components for the second stage and
PROSTALAC as a spacer: the infection-free rate was $89.7 \%$ at a minimum of two years follow-up.

Industrial production of an antibiotic-loaded PMMA spacer gives well-defined mechanical and pharmacological properties to the device. This ensures a reduction of mechanical failure and a standardized and known antibiotic release, compared with hand-made spacers. Moreover, it could reduce the surgical time. The disadvantage reported is that it is impossible to use the sensible drugs, because they are ready to use in the operating theatre.

They now offer a wide range of different head sizes in order to have a perfect couple with acetabulum, and different length of the stem. In a large case series, Romanò et al. [41] reported on 102 consecutive patients with infected THA treated by two-stage revision: 60 received a short-stem premade spacer (group S) and 42 a long-stem (group L). Systemic toxicity and spacer breakage were not observed. No difference was observed with regards to infection recurrence (one in group L, none in group S), spacer cranial dislocation (20\% in group L, $14 \%$ in group S) and HHS improvement. The infection-free rate overall was $96.1 \%$ at a mean follow-up of four years.

In a retrospective review, Hsieh et al. [42] compared the use of antibiotic-loaded cement beads to a preformed spacer. The infection-free rate was $95.3 \%$ (122 of 128 patients) being similar in both groups. The use of an articulating spacer was associated with a higher HHS, a shorter hospital stay and better walking capacity in the interim period. Additionally, at revision the articulating spacer group demonstrated decreased operative time, less blood loss and a lower transfusion requirement. There were fewer postoperative dislocations.

\section{OUR EXPERIENCE WITH TWO-STAGE REVISION USING AN INDUSTRIAL SPACER (SPACER G)}

Between 1999 and 2010, 30 patients (17 men and 13 women) with infected THA were treated with two-stage implant revision using a preformed antibiotic spacer. At the time of surgery, the mean age of patients was 71.4 years (range 54-84 years). The initial diagnosis was osteoarthritis in 20 cases, hip dysplasia in four, femoral neck fracture in four, femoral head necrosis in one, and rheumatoid arthritis in one. Nine of these patients had undergone a previous hip revision surgery due to aseptic loosening, recurrent dislocation, or periprosthetic fracture. The diagnosis of infection was based on clinical presentation and haematological parameters $([E S R] \geq 30 \mathrm{~mm} / \mathrm{h}$ and $[C R P] \geq 10$ $\mathrm{mg} / \mathrm{l}$ ), integrated with radiographic presentation (X-ray in two views, and bone scan with labelled leukocytes, Fig. 3a, b) and the presence of a draining sinus even in absence of a microbiological test. A bone scan with labelled leukocytes indicated an infection in all cases.

A posterior-lateral approach was used for surgical exposure of the infected implants.

The therapeutic protocol included a first procedure with prosthetic components removal, accurate debridement of infected and devitalized tissues, removal of any cement residues, and implantation of a preformed antibiotic spacer, Spacer-G/InterSpace (Tecres, Sommacampagna, Verona, 
(a)

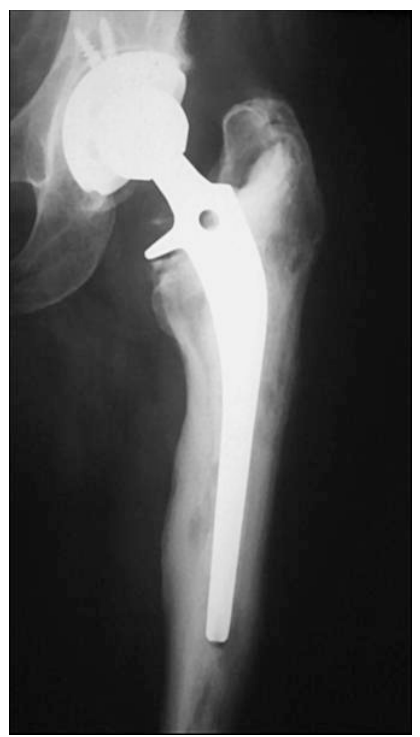

(b)

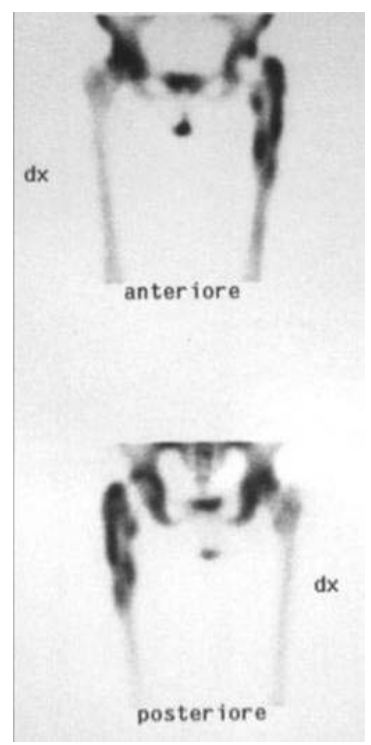

Fig. (3). Preoperative view of an infected THA sustained by Staphylococcus aureus (a), Labelled leukocyte scan revealed the site of infection (b).

Italy - Fig. 4). This is a preformed spacer, with a central load-bearing cylindrical rod made of stainless steel and encased in gentamicin-impregnated acrylic cement (1.87 $\mathrm{w} / \mathrm{w}$ in the polymerized resin, $3.2 \mathrm{w} / \mathrm{w}$ in the powder component) and available in three head sizes (46, 54, 60 $\mathrm{mm}$ ) and two stem lengths (standard and long).

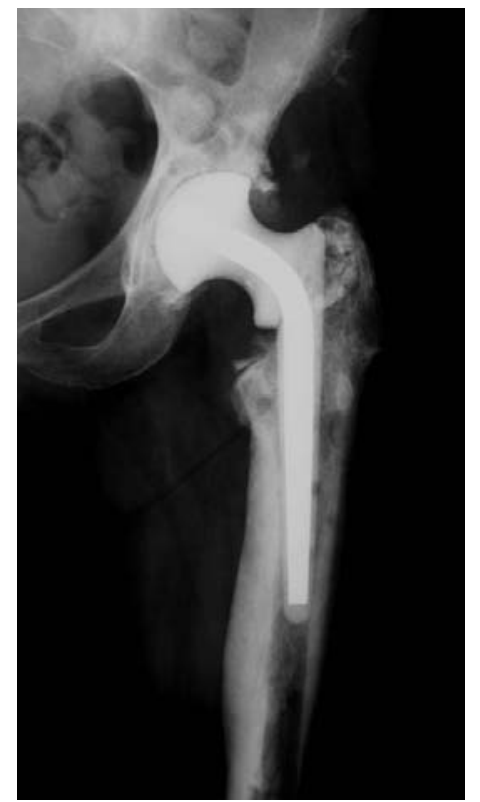

Fig. (4). AP view of the Spacer-G/InterSpace implanted.

At the time of explantation, multiple samples for culture were taken from joint fluid and granulation tissues at acetabular and femoral canal sites. After sampling, a wide spectrum intravenous antibiotic therapy was started until organism sensitivity tests were available, then the antibiotic therapy was tailored to the sensitivity of the bacterial strains. Infection was caused by Staphylococcus aureus (eight cases), Staphylococcus epidermidis (5 cases), Escherichia coli (2 cases), Streptococcus agalactiae (1 case), and

Streptococcus mitis (1 case); infection was polymicrobial in five cases. In eight patients bacteria were not identified and infection diagnosis was based on the presence of a draining sinus. In cases of unidentified microbiological agent a wide spectrum antibiotic, effective on the most common bacterial strains in our nosocomial environment, was chosen.

The spacer was left in situ for an average time of 5.5 months (range 1-13 months). During this period patients were allowed to walk with partial weight bearing, active and passive exercise, and muscle training were also encouraged. The patients were evaluated monthly with radiographs, and every 15 days white cells count (WCC), ESR, and CRP were tested. Systemic antibiotics were given until the serological markers were normalized for 2 weeks consecutively. After an additional month, ESR and CRP were re-tested and, if normal, the second-stage surgical procedure was scheduled.

The second stage consisted of removal of the spacer and implantation of a definitive prosthesis. Intraoperative tissue samples from joint fluid, acetabulum, and femoral canal were also obtained during the procedure. Twenty-three hemispherical cups and six revision cups were used, while 11 primary stems (five uncemented and six cemented) and 18 revision stems were used on the femoral side (Fig. 5a, b). One patient underwent a Girdlestone resection arthroplasty. After surgery, patients underwent a further cycle of antibiotic treatment (5 weeks, range 1-16 weeks) based on previous sensitivity tests. (a)

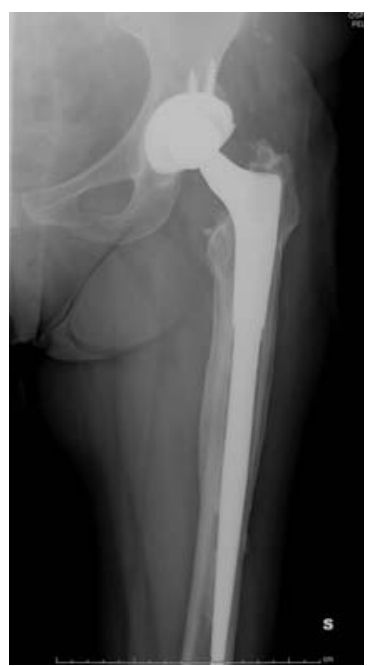

(b)

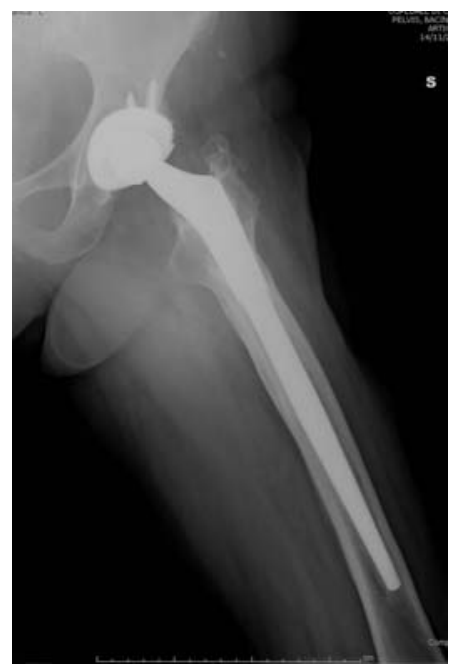

Fig. (5). AP and lateral view of the revision prosthesis at 8-year follow-up (a, b).

The patients' clinical records provided information on clinical evaluation before surgery (HHS, range of motionROM), duration of surgery, and period of hospitalization. At follow-up clinical (HHS, ROM), laboratory and radiographic data were recorded in order to evaluate and check pain control, infection eradication, functional outcomes, and eventual complications. Statistical analysis was performed using paired Student t-test $(\mathrm{P}<0.05)$.

The mean follow-up was of 72 months after the secondstage surgery (range 18-130 months), by evaluation of clinical and laboratory tests. Signs suggestive of deep infection were not observed in 29 out of 30 patients. The X- 
rays did not show any radiolucent lines, focal osteolysis, or component loosening. Recurrence of infection was observed in 1 female patient (Staphylococcus aureus) in which two draining sinuses were observed 20 days after spacer implantation. In this case, a Girdlestone resection arthroplasty was performed, because of her poor general health. The average hospital stay after the first stage was 13 days (range 7-28 days). Mean duration of first surgery was $138 \mathrm{~min}$ (range 60-180 min) with a mean blood loss of 1,750 $\mathrm{ml}$ (range 500-3,000 ml), while mean duration of second surgery was $127 \mathrm{~min}$ (range 55-210 min) with a mean blood loss of 1,690 ml (range 500-4.000 ml). In three cases, culture tests on samples taken during second stage were positive for infection but the identified microorganisms were not the same isolated during the first stage and were characterized by a low virulence and bacterial load; therefore they were considered contaminants.

Complications were observed in five patients: three spacer dislocations, one distal femoral fractures occurring during initial stem removal, and one femoral artery pseudoaneurysm, requiring stent placement.

Before treatment, the mean HHS was 43 (range 13-77); while before the second surgical procedure, the mean HHS was 51.4 (range 24-73). At final follow-up, the mean HHS was 82 (range 35-96). The difference between the initial and the final values was statistically significant according to Student t-test $(\mathrm{P}=0.001)$. At final follow-up, no dislocation or loosening of the definitive implants was observed.

\section{CONCLUSIONS}

Based on the most recently systematic review there is no suggestion that one- or two-stage revision methods have different re-infection outcomes [4]. In spite of promising results it has been demonstrated that one-stage direct exchange protocols using cemented and cementless implants, remains suitable for patients respecting previous described criteria. Anyway, long-term results are needed to establish the true durability of this approach [31].

The two-stage implant-exchange protocol remains the gold standard, considered as the most efficacious clinical approach for the treatment of periprosthetic infection, especially in patients with sinus tracts, swelling, extended abscess formation in depth and infection of MRSA, and other multidrug-resistant bacteria as reported in a recent consensus document [43].

\section{CONFLICT OF INTEREST}

The authors confirm that this article content has no conflict of interest.

\section{ACKNOWLEDGEMENTS}

Declared none.

\section{REFERENCES}

[1] Kurtz S, Ong K, Lau E, Mowat F, Halpern M. Projections of primary and revision hip and knee arthroplasty in the United States from 2005 to 2030. J Bone Joint Surg Am 2007; 89: 780-5.

[2] Puolakka TJ, Pajamaki KJ, Halonen PJ, et al. The Finnish Arthroplasty Register: report of the hip register. Acta Orthop Scand 2001; 72: 433-41.

[3] Lucht U. The Danish Hip arthroplasty register. Acta Orthop Scand 2000; 71: 433-9
[4] Beswick AD, Elvers KT, Smith AJ, Gooberman-Hill R, Lovering A, Blom AW. What is the evidence base to guide surgical treatment of infected hip prostheses? Systematic review of longitudinal studies in unselected patients. BMC Med 2012; 10(1): 18 .

[5] Berbari EF, Hanssen AD, Duffy MC, et al. Risk factors for prosthetic joint infection: case-control study. Clin Infect Dis 1998; 27: $1247-54$

[6] Cordero-Ampuero J, de Dios M. What Are the Risk Factors for Infection in Hemiarthroplasties and Total Hip Arthroplasties? Clin Orthop Relat Res 2010; 468: 3268-77.

[7] Engesæter LB, Espehaug B, Lie SA, Furnes O, Havelin LI. Does cement increase the risk of infection in primary total hip arthroplasty? Revision rates in 56,275 cemented and uncemented primary THAs followed for $0-16$ years in the Norwegian Arthroplasty Register. Acta Orthop 2006; 77(3): 351-8.

[8] Gomez E, Patel R. Laboratory Diagnosis of Prosthetic Joint Infection, Part I. Clin Microbiol Newslett 2011; 33: 8.

[9] Piper KE, Fernandez-Sampedro M, Steckelberg KE, et al. Creactive protein, erythrocyte sedimentation rate and orthopedic implant infection. PLoS ONE 2010; 5: e9358

[10] Di Cesare PE, Chang E, Preston CF, Liu CJ. Serum interleukin-6 as a marker of periprosthetic infection following total hip and knee arthroplasty. J Bone Joint Surg Am 2005; 87: 1921-7.

[11] Peel TN, Buising KL, Choong PFM. Prosthetic joint infection: challenges of diagnosis and treatment. ANZ J Surg 2011; 81(1-2): 32-9.

[12] Mc Bride TJ, Prakash D. How to read a postoperative total hip replacement radiograph. Postgrad Med J 87: 101-9.

[13] Love C, Tronco GG, Yu AK, et al. Diagnosing lower extremity (LE) prosthetic joint infection: Bone, gallium \&labeled leukocyte imaging. Presented at the 2008 SNM Meeting, New Orleans, LA, June 14-18, 2008.

[14] Chryssikos T, Parvizi J, Ghanem E, Newberg A, Zhuang H, Alavi A. FDG-PET Imaging can diagnose periprosthetic infection of the hip. Clin Orthop Relat Res 2008; 466: 1338-42.

[15] Love C, Marwin SE, Palestro CJ. Nuclear medicine and the infected joint replacement. Semin Nucl Med 2009; 39: 66-78.

[16] Klouche S, Lhotellier L, Mamoudy P. Infected total hip arthroplasty treated by an irrigation-debridement/component retention protocol. A prospective study in a 12-case series with minimum 2 years' follow-up. Orthop Traumatol Surg Res 2011; 97: 134-8.

[17] Byren I, Bejon P, Atkins BL, et al. One hundred and twelve infected arthroplasties treated with 'DAIR' (debridement, antibiotics and implant retention): antibiotic duration and outcome. J Antimicrob Chemother 2009; 63: 1264-71.

[18] Zimmerli W, Trampuz A, Ochsner PE. Prosthetic-joint infections. N Engl J Med 2004; 351: 1645-54.

[19] Klouche S, Leonard P, Zeller V, et al. Infected total hip arthroplasty revision: One- or two-stage procedure? Orthop Traumatol Surg Res 2012; 98(2): 144-50.

[20] Langlais F. Can we improve the results of revision arthroplasty for infected total hip replacement? J Bone Joint Surg Br 2003; 85(5): 637-40.

[21] Miley GB, Scheller AD, Turner RH. Medical and surgical treatment of the septic hip with one-stage revision arthroplasty. Clin Orthop Relat Res 1982; 170: 76-82.

[22] Buchholz HW, Elson RA, Heinert K. Management of deep infection of total hip replacement. J Bone Joint Surg Br 1981; 63: 342-53.

[23] Raut VV, Siney PD, Wroblewski BM. One-stage revision of total hip arthroplasty for deep infection. Clin Orthop Relat Res 1995; 321: 202-7.

[24] Jackson WO, Schmalzried TP. Limited role of direct exchange arthroplasty in the treatment of infected total hip replacements. Clin Orthop Relat Res 2000; 381: 101-5.

[25] Yoo JJ, Kwon YS, Koo KH, Yoon KS, Kim YM, Kim HJ. Onestage cementless revision arthroplasty for infected hip replacements. Int Orthop 2009; 33: 1195-201.

[26] Rudelli S, Uip D, Honda E, Lima ALLM. One-stage revision of infected total hip arthroplasty with bone graft. J Arthroplasty 2008; 23: 1165-77.

[27] Hanssen AD, Spangehl MJ. Practical applications of antibioticloaded bone cement for treatment of infected joint replacements Clin Orthop Relat Res 2004; 427: 79-85. 
[28] Fink B. Revision of late periprosthetic infections of total hip endoprostheses: pros and cons of different concepts. Int J Med Sci 2009; 6(5): 287-95.

[29] Winkler H, Stoiber A, Kaudela K, Winter F, Menschik F. One stage uncemented revision of infected total hip replacement using cancellous allograft bone impregnated with antibiotics. J Bone Joint Surg Br 2008; 90: 1580-5.

[30] Anagnostakos K, Furst O, Kelm J. Antibiotic-impregnated PMMA hip spacers: current status. Acta Orthop 2006; 77(4): 628-37.

[31] Senthi S, Munro JT, Pitto RP. Infection in total hip replacement: meta-analysis. Int Orthop 2011; 35(2): 253-60.

[32] Kelm J, ThiloRegitz, Schmitt E, Jung W, Anagnostakos K. In vivo and in vitro studies of antibiotic release from and bacterial growth inhibition by antibiotic-impregnated polymethylmethacrylate hip spacers. J Antimicrob Agents Chemother 2006; 50(1): 332-5.

[33] Duncan CP, Beauchamp CP. A temporary antibiotic loaded joint replacement system for management of complex infections involving the hip. Orthop Clin North Am 1993; 24(4): 751-9.

[34] Younger ASE, Duncan CP, Masri BA, McGraw RW. The outcome of two-stage arthroplasty using a custom-made interval spacer to treat the infected hip. J Arthroplast 1997; 12(6): 615-23.

[35] Klouche S, Sariali E, Mamoudy P. Total hip arthroplasty revision due to infection: a cost analysis approach. Orthop Traumatol Surg Res 2010; 96: 124-32.

[36] Jung J, Schmid NV, Kelm J, Schmitt E, Anagnostakos K. Complications after spacer implantation in the treatment of hip joint infections. Int J Med Sci 2009; 6(5): 265-73.

[37] Cabrita H, Camargo O, Lucia Lima A, Croci A. Prospective study of the treatment of infected hip arthroplasties with or without the use of an antibiotic loaded cement spacer. J Bone Joint Surg Br 2010; 92-B(Supp II): 314.

[38] FDA US Food and Drug Administration-Center for Device and Radiological Health (2001) In: Summary of safety and probable benefit. Available from: http://www.accessdata.fda.gov/cdrhdocs/pdf/H 00000 4b.pdf

[39] Scharfenberger A, Clark M, Lavoie G, O'Connor G, Masson E, Beaupre LA. Treatment of an infected total hip replacement with the PROSTALAC system. Part 1: infection resolution. Part 2: Health-related quality of life and function with the PROSTALAC implant in situ. Can J Surg 2007; 50: 24-33.

[40] Masri BA, Panagiotopoulos KP, Greidanus NV, Garbuz DS, Duncan CP. Cementless two-stage exchange arthroplasty for infection after total hip arthroplasty. J Arthroplasty 2007; 22: 72-8.

[41] Romanò CL, Romanò D, Logoluso N, Meani E. Long-stem versus short-stem preformed antibiotic-loaded cement spacers for twostage revision of infected total hip arthroplasty. Hip Int 2010; 20 (1): 26-33.

[42] Hsieh PH, Shih CH, Chang YH, Lee MS, Shih HN, Yang WE. Two-stage revision hip arthroplasty for infection: comparison between the interim use of antibiotic-loaded cement beads and a spacer prosthesis. J Bone Joint Surg Am 2004; 86: 1989-97.

[43] Leone S, Borre' S, d'ArminioMonforte A, and the GISIG (GruppoItaliano di Studio sulleInfezioniGravi) Working Group on Prosthetic Joint Infections. Consensus document on controversial issues in the diagnosis and treatment of prosthetic joint infections. Int J Infect Dis 2010; 14S4: S67-77.

(c) Cherubino et al.; Licensee Bentham Open.

This is an open access article licensed under the terms of the Creative Commons Attribution Non-Commercial License (http://creativecommons.org/licenses/by-nc/3.0/) which permits unrestricted, non-commercial use, distribution and reproduction in any medium, provided the work is properly cited. 